\title{
From mobility patterns to behavioural change: leveraging travel behaviour and personality profiles to nudge for sustainable transportation
}

\author{
Evangelia Anagnostopoulou ${ }^{1}$. Jasna Urbančič ${ }^{2}$. Efthimios Bothos ${ }^{1}$ (D) . \\ Babis Magoutas ${ }^{1} \cdot$ Luka Bradesko $^{2}$. Johann Schrammel ${ }^{3}$. Gregoris Mentzas ${ }^{1}$
}

Received: 1 October 2017 / Revised: 10 September 2018 / Accepted: 12 September 2018 /

Published online: 2 October 2018

(C) The Author(s) 2018

\begin{abstract}
Rendering transport behaviours more sustainable is a pressing issue of our times. In this paper, we rely on the deep penetration of mobile phones in order to influence citizens' behavior through data-driven mobility and persuasive profiles. Our proposed approach aims to nudge users on a personalized level in order to change their mobility behavior and make more sustainable choices. To achieve our goal, first we leverage pervasive mobile sensing to uncover users' mobility patterns and use of transportation modes. Second, we construct users' persuadability profiles by considering their personality and mobility behavior. With the use of the aforementioned information we generate personalized interventions that nudge users to adopt sustainable transportation habits. These interventions rely on persuasive technologies and are embedded in a route planning application for smartphones. A pilot study with 30 participants using the system for 6 weeks provided fairly positive evaluation results in terms of the acceptance of our approach and revealed instances of behavioural change.
\end{abstract}

Keywords Behavioural change $\cdot$ Mobility patterns $\cdot$ Personality $\cdot$ Data-driven profile Sustainable transportation

\section{Introduction}

Current transport systems suffer from a number of intractable problems, including congestion, emissions of greenhouse gases and local air pollutants, and accidents. Indeed, transport is the sector with the highest increase of greenhouse gas emissions in recent decades, and nearly one-third of global $\mathrm{CO}_{2}$ emissions come from the transportation of people. In addi-

Efthimios Bothos

mpthimios@gmail.com

1 ICCS of NTUA, National Technical University of Athens, Athens, Greece

2 Artificial Intelligence Laboratory, Jozef Stefan Institute, Ljubljana, Slovenia

3 AIT - Austrian Institute of Technology, Vienna, Austria 
tion, transport systems have significant impacts on the environment, accounting for between $20 \%$ and $25 \%$ of world energy consumption and carbon-dioxide emissions and road transport is a major contributor to local air pollution and smog. Given these problems, and their associated economic, social and environmental impacts, there is an emergent need for sustainable transportation.

Sustainable transport systems make positive contributions to the environmental, social and economic sustainability of the communities they serve. The available strategies for improving the sustainability of mobility vary and span from actions that affect the supply of transport to actions that affect the demand side. Regarding the supply side, indicative actions include investments on infrastructures or financial measures to reduce or limit the usage of vehicles that have lower levels of sustainability. Actions on the demand side involve making people aware of the negative impacts of their transport choices and nudging them towards the use of sustainable transport modes. One way to achieve this is to design and implement approaches that increase travelers' awareness of the environmental impact of travel mode choices and provide behavioral change interventions towards adopting transportation habits that reduce single occupancy vehicles and rely more on the use of public transportation, bicycles and walking than on private cars.

In this paper, we rely on the deep penetration of smartphones in order to influence citizens' behavior through data-driven mobility and persuasive profiles. Our proposed approach aims to nudge users on a personalized level to change their mobility behavior and make more sustainable choices. For this purpose, our work relies on the concept of persuasive technologies which have been successfully utilized in a number of application domains such as health, education and environmental awareness including environmental sustainability to promote greener transportation habits (see e.g., Wiafe and Nakata 2012). Persuasive technologies (Fogg 2002) refer to the application of psychological principles of persuasion (such as credibility, trust and reciprocity) to interactive media, with the aim to change users' attitudes and behaviors. They deliberately attempt to infuse a cognitive and/or an emotional change in the mental state of a user to transform the user's current cognitive state into another planned state (Torning and Oinas-Kukkonen 2009). On the level of design, persuasive technologies guide users towards the adoption of desired attitudes or actions by infusing persuasive strategies in applications that interact with the users. Persuasive strategies may consist of primary task support (i.e., features supporting the core activity or behavior), computer-human dialogue support (i.e., feedback from the system), perceived system credibility (i.e., features making the system seem credible and trustworthy), and social influence (i.e., features inducing motivation through social influence) (OinasKukkonen 2013). Detailed design guidelines and principles for these elements are presented in Oinas-Kukkonen and Harjumaa (2008).

With our approach, we aim to understand travelers' behavior, use this information as the basis for generating recommendations that correspond to their needs and nudge travelers to make sustainable mobility choices. More specifically, we focus on the following research questions: i) How to leverage pervasive mobile sensing to uncover users' mobility patterns and usage of transportation modes? We investigate machine learning algorithms that process GPS and accelerometer data for travel mode detection and provide extensions for identifying travel patterns and mode changes. ii) How to construct users' persuadability profiles, i.e. profiles that capture user's susceptibility to different persuasive strategies, by considering their personality and mobility behavior? We rely on users' personality and mobility type in order to define persuadability profiles. iii) How to use the aforementioned information to generate personalized interventions that nudge users to adopt sustainable transportation 
habits? These interventions rely on persuasive technologies and are embedded in a route planning application for smartphones. More specifically we focus on properly structuring the available route options to reach a destination and present persuasive messages that urge users to follow routes that are sustainable. Our application relies on sets of "multimodal" routes, i.e. routes that involve combinations of transportation modes (e.g. using a combination of a personal car and public transportation or using car and bike sharing services to reach a destination).

The remainder of the paper is organized as follows. Section 2 presents the related work and shows how our approach for sustainable mobility differs from existing ones; Section 3 provides an overview of our approach and explains its main assumptions and elements; Section 4 describes our methods for generating mobility profiles based on users' mobility patterns; Section 5 presents our methods for generating persuadability profiles with the use of information from users' personality and mobility type; Section 6 describes how we make use of these profiles and other related information, including contextual elements in order to infuse personalized behavioral change support in route planning applications; Section 7 provides the results and related discussion of a real life pilot where users from the city of Vienna used a route planning application which integrates our approach for a period of 6 weeks; Finally, Section 8 concludes the paper with our final remarks and suggestions for future work.

\section{Related work}

Persuasive systems addressing behaviour change in the context of personal mobility in urban environments is an active area of research, and numerous systems and implementations exist, aiming to motivate users towards making more eco-friendly choices; i.e. adopting transportation habits that rely more on the use of public transportation, bicycles and walking, and less on private cars. One of the first attempts was the PerCues mobile app published by Reitberger et al. (2007) which aimed to persuade people to use public transportation instead of their car in order to reduce emissions. The approach was based on displaying bus and pollution information, such as the departure time of the next bus and the decrease in emissions achieved by taking the bus instead of the car. Another way existing systems use to nudge users is by providing visual feedback in the form of adapting the background graphics of the smartphone when making sustainable choices. A prominent example is the UbiGreen app proposed by Froehlich et al. (2009) that encouraged greener alternatives, including carpooling, public transport and pedestrian modalities by providing visual feedback when users reduced driving. UbiGreen sensed users' behaviour and fed it back as ambient changes to the background graphics of the user's phone. The Peacox app, published by Bothos et al. (2014), influenced urban travellers to consider the environmental friendliness of travel modes while planning a route. Peacox embeds $\mathrm{CO}_{2}$ emissions visualizations as well as personal and collaborative challenges aiming to persuade users to reduce the emissions caused by their mobility choices.

Message based persuasive technologies have been implemented in various domains with promising results. Most of the previous work focuses on the health domain where indicative applications include motivating adherence to insulin therapy in adolescent diabetics (Franklin et al. 2006), smoking cessation (Rodgers et al. 2005), increasing physical activity (Purpura et al. 2011). Common persuasive strategies instantiated through messages include praise, reminders and suggestion. Messages praising the user are provided in response to a 
goal being achieved or in response to user data captured by the application. After successfully responding to a prompt by performing a goal, a user might receive a message praising their accomplishment (Bond et al. 2014). Reminders are commonly implemented as periodic messages sent to the user reminding them to check the application. For instance, in (Harries et al. 2013) generic daily or weekly reminders were sent encouraging users to continue using the application for improving their physical activity. Moreover, reminders were sent to user who did not meet their goals in order to maintain the motivation of the user (Hong et al. 2013). Suggestions entail messages recommending a particular behaviour to be carried out. Suggestion also comes in the form of motivational text that provides tips for continuing a specific behaviour (Fukuoka et al. 2011). In the transportation domain, the most relevant application integrating persuasive messages has been suggested by Bothos et al. (2014) in an application that nudged users to shift to less polluting transportation modes.

Most persuasive applications employ a one-size-fits-all approach to the delivery of persuasive attempts, failing to deliver personalized persuasion that leverages user characteristics and preferences, or to provide personalized tools that assist users in achieving the intended goals. For example, feedback based approaches provide a generic type of feedback (e.g. $\mathrm{CO}_{2}$ emissions) to all users and goal-setting based approaches provide the same goals to all users. Furthermore, none of the existing applications consider differences in users' susceptibility to persuasive strategies. However, user-adaptive systems can be more effective and/or usable than non-adaptive systems and personalized persuasive applications can have greater impact in transport behaviour change. In our work, we take into account data-driven user profiles containing the mobility patterns of travellers, their personality and mobility type in order to provide adaptive and personalized message based persuasion. Our approach personalizes the persuasive strategies employed by identifying users' susceptibility on specific strategies and continuously adopts them based on users' response to these strategies.

\section{Our approach for sustainable transportation}

Our aim is to support sustainable transportation decisions by providing targeted behavioural change interventions through data driven user profiles. Users are persuaded to select more environmentally friendly routes with tailored interventions on the basis of their characteristics, contextual, routing, mobility patterns and other relevant information. For this purpose, we have designed and implemented an approach that is integrated in a route planning mobile application developed as part of the OPTIMUM project ${ }^{1}$. The conceptual architecture of our approach is presented in Fig. 1.

Travellers who want to move from point A to point B issue route requests through the mobile application and receive a list of alternative unimodal and multimodal route options to choose from (section 1 of Fig. 1). Note that unimodal options involve the use of one transportation mode (e.g. public transportation or car) whereas multimodal options involve the use of two or more transportation modes (e.g. a combination of public transportation and bike sharing).

The route results are generated by an external routing engine with capability to generate multimodal route alternatives (section 2 of Fig. 1). The engine identifies the available mode combinations and routes which can be used to reach a destination. Once these results are calculated, our persuasive interventions services process them and attach persuasive cues in the form of persuasive messages (section 5 of Fig. 1). This processing is performed by two complementary services: the route recommendation service, and the persuasive messages 


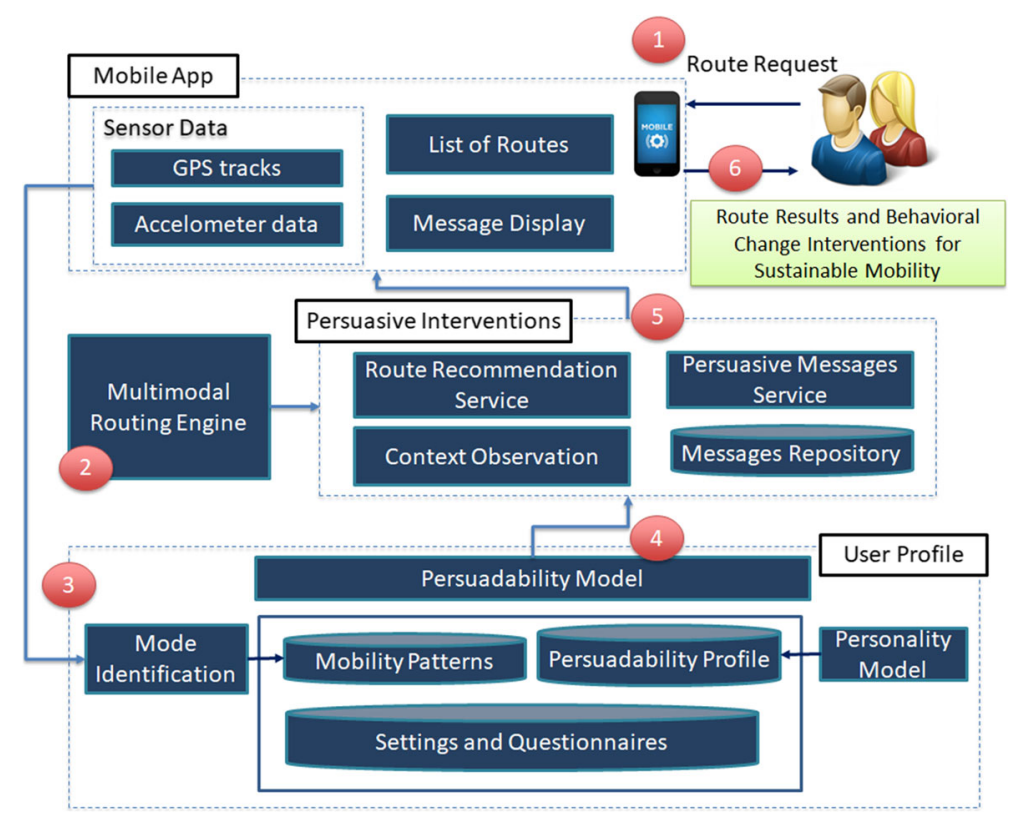

Fig. 1 Overview of our approach

service as shown in Fig. 1. The processes of tailoring the routes and the message based interventions to the specific user, make use of information stored in the user profile (section 4 of Fig. 1). This information includes: i) users' mobility patterns which are inferred from a mode identification function by analysing accelerometer data (section 3 of Fig. 1) and ii) a dynamic persuadability model which is able to adapt the employed persuasive strategies to the profile and behaviour of individual users. The latter adaptation is performed by monitoring the effect of the persuasive strategies and users' personality characteristics. In the following sections, we analyse the building blocks of our approach.

\section{Mobility profiles based on mobility patterns}

Ever since smartphones appeared and gained widespread adoption there have been many research efforts for their use in activity recognition and transportation mode detection. While the first attempts to recognize user activity were initiated before smartphones, the real effort in that direction begun with the development of mobile phones having built-in sensors (Reddy et al. 2010), including GPS and accelerometer sensors. Although GSM triangulation and local area wireless technology (Wi-Fi) can be employed for the purpose of transportation mode detection, their accuracy is relatively low compared to GPS (Shafique and Hato 2015) so latest state of the art research is focused on transportation mode detection based on GPS tracks and/or accelerometer data. Approaches that rely solely on GPS trajectories require GPS signal of high quality, but the GPS receivers of smartphones are generally severely shielded during daily activities (Widhalm et al. 2012). This occurs during underground travels, inside stations, or even when users are not sufficiently close to a window when travelling in a vehicle (Hemminki et al. 2013). In all of these cases the result is 
loss of positioning information. Another known issue when using GPS signals from mobile devices is the high power consumption (Hemminki et al. 2013), which is especially not pleasing in case of longer commutes. Both of these two issues suggest that accelerometer sensor data can be more appropriate for activity detection. There have also been successful attempts of using other smartphone sensors, for example magnetometer (Chen et al. 2017) and microphone (Lee et al. 2017) in combination with accelerometer data.

Machine learning methods that are most commonly used in accelerometer based mode detection include support vector machines, decision trees and random forests, although naïve Bayes, Bayesian networks and neural networks have been used as well (Shafique and Hato 2015). Often these classifiers are used in an ensemble (Widhalm et al. 2012). The majority of algorithms use Adaptive Boosting or Hidden Markov Model to improve the performance of the methods mentioned above (Reddy et al. 2010; Shafique and Hato 2015; Widhalm et al. 2012; Hemminki et al. 2013).

Features used for machine learning can be divided into 5 groups: Statistical, Time, Frequency, Peak and Segment (Hemminki et al. 2013), however in most cases statistical features and features based in frequency are used (Reddy et al. 2010; Shafique and Hato 2015; Widhalm et al. 2012). Previous work has shown that extracted features from accelerometer data are able to capture the characteristics of high-frequency motion caused by user's physical movement, vehicle's engine, and contact between wheels and surface (Hemminki et al. 2013). These characteristics allow machine learning classification algorithms, and through proper training, to differentiate cars and buses even though they share the roads. Similarly, driving a car or travelling in a car as a passenger should not affect the classification.

Our approach focuses on near real-time classification of motorized transportation modes. We are trying to preserve battery life by using short samples of accelerometer readings, which also reduces the consumption of mobile data, although our system can also successfully operate without having the mobile data option turned on. We opted for a computationally inexpensive classification algorithm as we desire to move sample classification from the server to the phone in order to save additional resources.

\subsection{The mobility patterns transportation mode detection module}

User mobility patterns are identified through a GPS tracking mobile library for Android and iOS mobile devices, named "mobility patterns". The library runs in the background and collects GPS tracks of users' movement. GPS coordinates are then sent to a server, where they are clustered to detect so called staypoints (locations, where user stayed for more than few minutes in a small radius) and paths that connect consecutive staypoints. Staypoints are interesting because they can be matched with places that the user might have visited previously or is visiting on daily basis, for example home, work, store, gym or bar. This information is retrieved from Foursquare or other similar databases. For geoclustering we explored multiple algorithms (Senožetnik et al. 2016), and at the end selected an improved version of the Stay Point Detection (SPD) algorithm (Kang et al. 2004) due to its implementation simplicity and computational performance. The core of the algorithm is temporal and spatial rule defining a staypoint as a set of the coordinates (of the user's mobile device) that all lay inside a given perimeter for at least minimum time:r(lat,lon) T_p t(lat,lon) T_t. This proves to be robust to the usual GPS signal, which is not always returning the same coordinates for the same location and is also lost during the time the user is indoor. The algorithm was extended to not discard the locations which are part of the paths, since we are not only interested in the visited locations but also in the movements between them. Additionally, the algorithm was extended with an additional pass ${ }^{2}$ which corrects the mistakes 
that can appear in the first pass, such as multiple consequent staypoints which are very close together and the paths between do not last more than a given threshold.

However, exploiting other mobile sensors in addition to GPS positioning is possible. Mobile operating system (OS) developers are aware of such possibilities, therefore their APIs include activity recognition packages. However, these packages detect only a limited set of modes which include: still, walking, running, cycling, and in vehicle. Such coarsegrained classification is not enough for tracking and routing purposes, especially in usecases for urban environments, where public transportation with buses and trains can be a good alternative to private vehicles.

Our mobility patterns module makes use of native OS APIs to get users' activities and appends the results to the GPS coordinates. If the APIs report that the user is travelling in vehicle, the mobility patterns module collects an accelerometer sample that is sent to the server together with the current coordinates and possible activities for further processing. We transform the information about probable activities into vector shape a for easier manipulation and combine activities $\vec{a}_{l}$ for all locations 1 in a path to get the most probable modality of this path using the following formula:

$$
\mathbf{a}=\frac{\sum_{l \in \text { path }} d_{l} \vec{a}_{l}}{\sum_{l \in \text { path }} d_{l}}
$$

where $\mathrm{d}_{l}$ is the distance between two consecutive GPS locations in the path. The theoretical background and the algorithm used for transportation mode classification are described in the following section.

\subsection{Algorithmic description}

This section provides a detailed description of (training) data acquisition, pre-processing and classification of motorized transportation modes (e.g. car, bus, train). For classification of non-motorized modes, such as walking, cycling or running we use native OS APIs as stated in the previous section. We also describe the evaluation of the training process, where we used 10-fold cross-validation to test our classifier. Whenever the phone's activity recognition system detects that the user is travelling in a vehicle, we collect a 5 s sample of accelerometer signal. We are using short samples in order to reduce the computation time and have faster response time for real-time classification. Additionally, this preserves battery life, saves space and reduces the usage of mobile data (Hemminki et al. 2013).

We collected the data for the initial model manually. This means that we developed a GPS tracking application that is based on the mobility patterns GPS tracking library, and extended it with the accelerometer measuring feature, so that the collection of the accelerometer readings begins and ends manually with the press of a button. Measurements were acquired by initiating the data collection just before travelling by car/bus/train/motorbike and stopping it at the destination. For the purposes of this manual data collection process, we paid a student to travel around the city of Ljubljana using different means of public transportation (e.g. bus, train), whereas car samples were collected by four members of our laboratory in order to account for different driving styles. We collected approximately $20 \mathrm{~h}$ of accelerometer measurements with travel modes distributed as follows: $57 \%$ car, $32 \%$ bus, $11 \%$ train, $0.1 \%$ motorbike.For the training process, we split the accelerometer recordings into $5 \mathrm{~s}$ samples as part of the pre-processing. Training data were stored in text files containing 4 columns including the timestamp of the measurement, and the accelerometer readings along 3 axes, $x, y$, and $z$. The mobility patterns module sends 
accelerometer samples in a JSON representation with one numeric field that contains the timestamp of the first accelerometer reading, and 3 array fields that contain accelerometer measurements along the 3 axes.

One problem we faced concerned the correlation of acceleration measurements with the orientation of the phone in the 3D space. Practically this means that gravity is measured together with the dynamic acceleration caused by phone movements and has to be subtracted. Thus, we have to be able to separate the constant acceleration caused by the gravity and the dynamic part of the acceleration. The gravity estimation algorithm works as follows: for a chosen time interval (in our case $1 \mathrm{~s}$ ), we obtain an estimate of the gravity component on each axis by averaging all measurements in the interval on that axis following the work of Mizell (2003). After obtaining the estimates, we subtract the gravity component from all of the entries on the corresponding axis in the given time interval. Through this we obtain only the dynamic acceleration component of the signal.

\subsubsection{Classification}

After pre-processing the accelerometer readings, we extract 60 features for the classification process. We use the mean, standard deviation, skewness, 5th, and 95th percentile of acceleration data on all three axes and overall amplitude. We also split the acceleration into a positive part, which indicates that the velocity of movement in that direction is increasing, and a negative part, which indicates that the velocity is decreasing. The five statistics mentioned above are calculated for these two parts plus the total acceleration, across the three axes plus the overall amplitude, summing up to 60 features.

For our classification we use support vector machines classifier (SVM) as similar work has shown quite successful results (Shafique and Hato 2015). The implementation of the classifier is based on the data analytics platform QMiner, an open source platform written in $\mathrm{C}++$ and exposed via JavaScript API, which is used for large scale data analysis (Fortuna et al. 2014). The SVM classifier (SVC) in QMiner was implemented as a binary classifier, so we focused on a binary classification of each modality. We trained the classifier in OneVS-All and One-VS-One manner and we found that in our case One-VS-One provides better results (Urbančič et al. 2016). In the One-VS-One approach we label samples from one class as positive and samples from another class as negative, whereas all other samples are filtered out. We used 10-fold cross-validation on training data to evaluate the training process and tune the hyperparameters of the model. We searched the space of possible values of learning parameters and opted for the combination that produced the highest average F1 score. The results of One-VS-One binary classification are presented in Table 1, where rows represent positive and columns negative samples. Table 1 shows that car and train modes are very well distinguishable from each other, whereas accuracy and F1 scores are slightly lower when recognizing buses compared to car and train classes.

Table 1 Accuracy/F1 score for One-VS-One binary classification

\begin{tabular}{lllr}
\hline Positive/Negative & Car & Bus & Train \\
\hline Car & & $0.848 / 0.889$ & $0.815 / 0.760$ \\
Bus & $0.856 / 0.832$ & & $0.858 / 0.896$ \\
Train & $0.934 / 0.883$ & $0.815 / 0.760$ & \\
\hline
\end{tabular}

Rows represent positive examples for classification, whereas in columns are negative labels 
Table 2 Confusion matrix for multiclass classification with SVM classifier from QMiner, and decision tree (DT) and random forest (RF) classifiers from Scikit-learn

\begin{tabular}{|c|c|c|c|c|c|c|c|c|c|}
\hline \multirow{2}{*}{$\begin{array}{l}\text { True/Predictive } \\
\text { Method }\end{array}$} & \multicolumn{3}{|l|}{ Car } & \multicolumn{3}{|l|}{ Bus } & \multicolumn{3}{|l|}{ Train } \\
\hline & SVM & DT & $\mathrm{RF}$ & SVM & DT & $\mathrm{RF}$ & SVM & DT & $\mathrm{RF}$ \\
\hline Car & 0.893 & 0.898 & 0.962 & 0.088 & 0.098 & 0.034 & 0.019 & 0.004 & 0.003 \\
\hline Bus & 0.282 & 0.138 & 0.221 & 0.573 & 0.848 & 0.719 & 0.145 & 0.015 & 0.060 \\
\hline Train & 0.167 & 0.157 & 0.140 & 0.162 & 0.355 & 0.177 & 0.671 & 0.488 & 0.682 \\
\hline
\end{tabular}

Once we identified that the binary classification was successful, we also implemented multiclass classification using the 6 binary classifiers that we had already trained earlier. We combined their predictions based on the distance between the separating hyper plane and the sample. We tested the performance of the classifier using 10-fold cross validation. We compared the performance of our SVM multiclass classifier to the performance of decision tree (DT) and random forest (RF) classifiers from Python's library Scikit-learn (Pedregosa et al. 2011). Table 2 contains the confusion matrix for multiclass classification with SVM classifier from QMiner, and decision tree (DT) and random forest (RF) classifiers from Scikit-learn. Values for SVM are in italics as SVM is our chosen classifier. On the diagonal we bolded the highest score achieved for that mode, whereas outside of the diagonal we bolded the lowest result. We can observe that for each mode the majority of instances is classified correctly, except for train when classifying with decision tree (underlined). In that case, more than half of train samples is miss-classified. This shows that decision tree often confuses trains for buses, which is not unexpected as we observed something similar in Table 1. In Table 1 we showed that we are able to distinguish trains from buses with F1 score of 0.76 . This is the lowest score among all combinations of modes. Random forest confuses the least modes as it has overall the lowest values outside the diagonal. SVM classifies the least train examples as buses, and decision tree makes the least mistakes in classifying bus instances as cars.

Table 3 contains values of different metrics that we used to test our classifier. We used italics for SVM classifier as it is our chosen method, whereas we bolded the highest scores for each metrics. The observation that buses and trains are harder to recognize is also noticeable in Table 3 as these modes achieve significantly lower precision, recall, and F1 scores than cars. Random forest scores the highest precision, recall, and F1 score on average, however the difference in precision between decision tree and random forest is not significant.

Table 3 Classification accuracy, precision, recall, and F1 score for multiclass classification

\begin{tabular}{|c|c|c|c|c|c|c|c|c|c|c|c|c|}
\hline \multirow{2}{*}{$\begin{array}{l}\text { True/ Predictive } \\
\text { Method }\end{array}$} & \multicolumn{3}{|c|}{ Accuracy } & \multicolumn{3}{|c|}{ Precision } & \multicolumn{3}{|l|}{ Recall } & \multicolumn{3}{|c|}{ F1 Score } \\
\hline & SVM & DT & $\mathrm{RF}$ & SVM & DT & $\mathrm{RF}$ & SVM & DT & $\mathrm{RF}$ & SVM & DT & $\mathrm{RF}$ \\
\hline Car & 0.825 & 0.876 & 0.861 & 0.785 & 0.859 & 0.831 & 0.893 & 0.897 & 0.962 & 0.835 & 0.878 & 0.908 \\
\hline Bus & 0.787 & 0.839 & 0.836 & 0.724 & 0.717 & 0.832 & 0.573 & 0.847 & 0.719 & 0.640 & 0.777 & 0.718 \\
\hline Train & 0.886 & 0.904 & 0.900 & 0.672 & 0.923 & 0.847 & 0.671 & 0.488 & 0.682 & 0.671 & 0.638 & 0.784 \\
\hline Average & 0.832 & 0.873 & 0.866 & 0.727 & 0.834 & 0.837 & 0.712 & 0.744 & 0.787 & 0.716 & 0.764 & 0.803 \\
\hline
\end{tabular}


Although results in Tables 2 and 3 reveal that our linear SVM classifier is not optimal for this problem, we opted for it because of the desired efficiency of the server, which we mentioned before. As random forest performed better than SVM we believe that using kernel functions and a better strategy to combine the results of multiple binary classifiers might help to boost the performance of SVM.

\section{Persuadability profiles based on personality and mobility type}

User persuadability refers to the user's susceptibility to different persuasive strategies. For the purposes of our work we focus on three main persuasive strategies adopted from Fogg (2002) which are supported by the data driven profiles generated through the mobility patterns approach described in Section 4. These strategies are self-monitoring (i.e. providing insights of one's behaviour over time), comparison (i.e. providing information related to how one's behaviour compares to that of peers) and suggestion (i.e. generating smart advices for sustainable behaviour). The strategies are delivered in the form of persuasive messages which are presented along with a set of route options. The aim of the presented persuasive message is to nudge the user to select a sustainable route choice.

We identify users' persuadability with respect to the three strategies mentioned above through a dynamic and implicit process, which means that there is no need of explicit user involvement. The process is dynamic and after a number of attempts to persuade the user, the system converges to the persuasive strategy that works best for the specific user. Our approach builds upon the work of Kaptein (2011) who used a Bayesian approach for adapting user persuasion profile based on user's interaction with an online e-commerce store. We adopted such an approach that considers previous successful persuasive interactions for a particular user and other similar users and extended it with the aim to address the problem of missing information for new users (cold start problem). Based on Kaptein (2011), the effectiveness of a single persuasive strategy (in our case implemented through messages as mentioned above) for a particular user is estimated by considering the specific user's previous responses to that same message and the known effectiveness of the message for other users. In our case, a persuasive interaction is considered to be successful when the user declares that the message affects her/his route choice. In order to acquire such feedback, we have implemented a small popup that is presented in the application and explicitly asks the user if the presented message affected her/his decision (see Section 6 for more details).

The probability of a user being persuaded on multiple occasions is regarded as a binomial random variable $B(n, p)$ where $n$ denotes the number of attempts to persuade the user by using a specific strategy and p denotes the probability of success, i.e. in our case is the probability of following the route on which the persuasive message is attached to. Given S different strategies, we compute for each individual and each strategy, the probability $\mathrm{p}=\mathrm{k} / \mathrm{n}$ where $\mathrm{k}$ is the number of observed successes after the presentation of the message implementing this strategy $n$ times to a specific user. Every time that the system displays a message to an individual, the probabilities of success of each strategy are updated and the user is considered more susceptible to the strategy that has the highest $\mathrm{p}$ value for her/him. The higher the number of users receiving persuasive interventions and the number of persuasive attempts per user, the faster this dynamic approach converges.

However, this approach suffers from the so-called cold start problem, according to which the system cannot draw any inferences for users until it has gathered sufficient information 
for them. To address this problem and enable faster convergence of the algorithm, we have implemented a model that is used to explicitly identify the initial user susceptibility to the different persuasive strategies based on users' personality and mobility type. This model has been presented in our previous work (Anagnostopoulou et al. 2017) and provides correlations of user's personality and mobility type with her/his susceptibility to the various persuasive strategies. In our work the definition of personality stems from the big five factor model, which measures a user's personality type in terms of five dimensions, namely Openness, Conscientiousness, Extraversion, Agreeableness and Neuroticism (also known with the acronym OCEAN) (Collis 1997). Each user's personality type is associated with certain characteristics. The measurement of users' personality type is performed using specific instruments. The best known for contexts in which users' time is limited, is the Big Five Inventory proposed by Rammstedt and John (2007). This instrument consists of ten 5-point Likert scale questions which are used to get a measure of the five personality dimensions. The questions are answered by each user during registration in the OPTIMUM application. User responses are used to estimate her/his five personality traits by inferring a score per personality trait. The trait with the highest score is the one that characterizes the user the most. That personality trait in turn feeds our persuadability model with the aim to initialize the individual user's persuadability profile.

Regarding the user mobility type, this refers to classifications of travellers into potential "mode switchers" and it's a concept that has been conceived in order to support mobility management policies. A widely-adopted classification of mobility types is provided by Anable (2005) and represents a unique combination of preferences, worldviews and attitudes, indicating that different groups need to be serviced in different ways to optimize the chance of influencing mode choice behaviours. The work of Anable proposes the following eight mobility types (in order of declining car dependence): Devoted Drivers, Image Improvers, Malcontented Motorists, Active Aspirers, Practical Travelers, Car contemplators, Public Transport Dependents and Car-free Choosers. In our work we use three main user mobility types, which group the aforementioned types in three categories: Drivers, i.e. people that actively use their car and do not wish to change this habit (a group including Devoted Drivers and Image Improvers), Potential non-Drivers, i.e. people that actively use their car but they want to switch to more environmentally friendly modes of transport (a group including Malcontented Motorists, Active Aspirers, Practical Travelers) and non-Drivers, i.e. people who do not use a car (a group including Car contemplators, Public Transport Dependents, Car-free Choosers).

The mobility type of users is inferred during registration in the OPTIMUM app where the user is asked to provide the transportation modes they use the most and their attitude towards other modes of transport. A ranked list of persuasive strategies that work best for users with different combinations of personalities and mobility types based on the persuadability model developed as part of our previous work (Anagnostopoulou et al. 2017) is the following: Drivers and Potential non-Drivers with high agreeableness scores: 1. Comparison, 2. Self-monitoring, 3. Suggestion; Potential non-Drivers with high neuroticism scores: 1 . Suggestion, 2. Comparison, 3. Self-monitoring; Non-Drivers with high neuroticism scores: 1. Self-monitoring, 2.Suggestion, 3.Comparison; Drivers with high Extraversion, Openness, neuroticism or Conscientiousness scores: 1. Comparison, 2. Self-monitoring, 3. Suggestion; Potential non-Drivers with high Extraversion, Openness or Conscientiousness scores: 1. Comparison, 2. Suggestion, 3. Self-monitoring; Non-Drivers with high Extraversion, Openness, agreeableness or Conscientiousness scores: 1. Self-monitoring, 2. Comparison, Suggestion. 


\section{Personalized behavioural change support}

Our personalized behavioural change support approach relies on two complementary services that structure the route results (i.e. the route recommendation service) and attach persuasive messages to these results (i.e. the persuasive messages service) (see also Fig. 1). The route recommendation service receives as input a list of alternative routes for travelling from origin A to destination $\mathrm{B}$. The list is generated by a routing engine and contains an extended set of unimodal and multimodal options for reaching the destination. The service integrates functionalities for filtering and structuring the available routes, and returns a list of recommended routes. Filtering concerns the exclusion of routes based on preferences set by the user in her/his profile and other pre-defined restrictions. More specifically users set if they own a car or bicycle and the maximum walking and biking distance they are willing to travel. System defined restrictions include the filtering of routes that commonly do not make sense (e.g. taking a car for $100 \mathrm{~m}$ ). Structuring involves ranking of the filtered routes in a personalized manner, and highlighting one route that is environmentally friendly and adheres to user preferences as well as the current context. A utility function has been defined for ranking the routes and selecting the one to be highlighted. It captures the effect of psychometric and demographic parameters on travel time and cost, user stated preferences, past user behaviour, active context variables and the environmental friendliness of the routes in terms of the emissions caused.

The highlighted route, which is displayed in a prominent position in the OPTIMUM application, is considered as the target for the persuasive attempts (see part 1 of Fig. 2 for an indicative capture of the routes presentation screen of the application). Given a route as

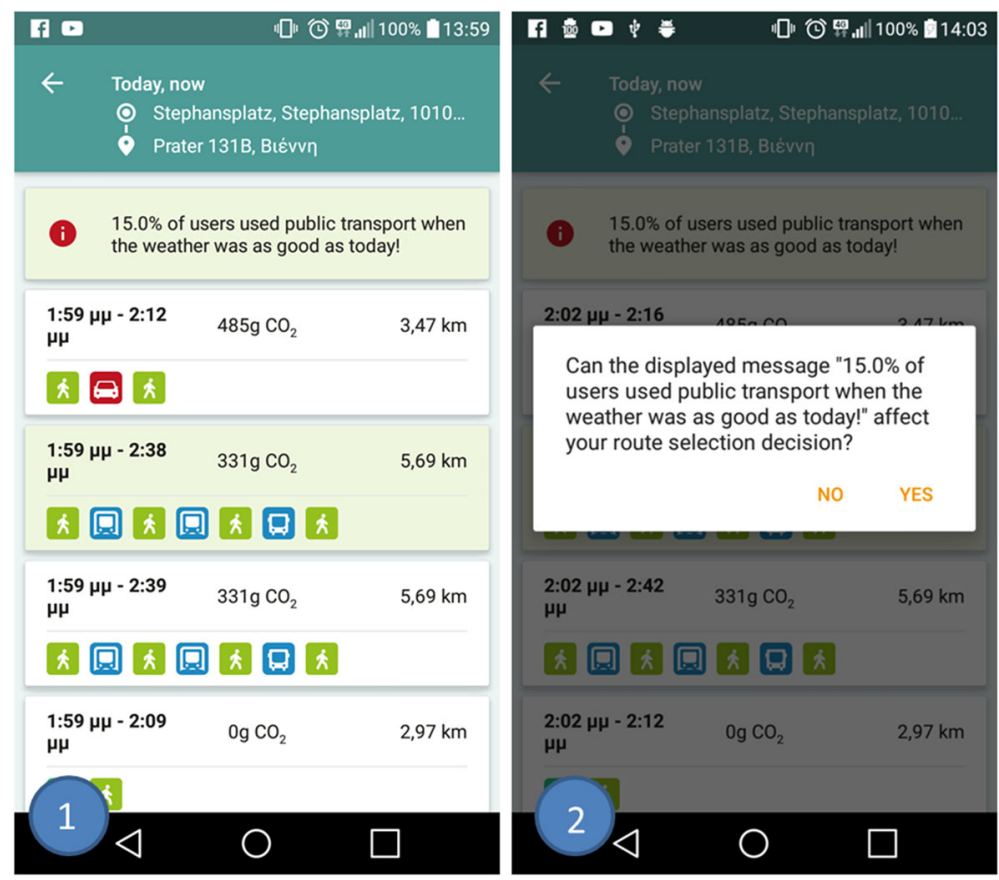

Fig. 2 Example of route results in Optimum app (part 1). A popup asks users to provide feedback (part 2) 
the target to nudge the user to follow, the role of the persuasive messages service is to provide personalized persuasive messages that aim to persuade the user to take that particular route. The persuasive messages service attaches a persuasive message to the targeted route for user persuasion. The service is personalized as its design is such that it considers that fact that users respond differently to the various persuasive strategies. The persuasive messages service specifically addresses different users and tailors the persuasive messages to the individual, with the aim to maximize the impact of the persuasive attempts. The service selects from a set of persuasive messages corresponding to different persuasive strategies, the message that each individual user is more susceptible to, by also considering the current user, trip and environmental context.

\subsection{Design of messages}

We designed 98 persuasive messages with each one implementing a single persuasive strategy. Multiple messages have been designed per persuasive strategy. The messages are context-aware thus enhancing the ability of the persuasive messages service to provide tailored messages. Only messages with a context that is valid for a particular user with a particular trip profile, who is planning for a particular trip made under specific environmental conditions, are selected. We have defined persuasive messages that are valid for seven different contextual variables which are described in the following.

Our approach for defining the context variables was based on the conceptual framework provided by Lamsfus et al. (2015). The framework considers that travel behaviour takes place in a context that consists of various aspects, including the personal characteristics, the trip-related characteristics and the environment domain, in a specific stage (time) of the travel process. The context variables are defined based on the above framework and are binary, which means that they are activated when the conditions that define them are present, and depend on the user's profile and recorded behaviour, the characteristics of the alternative routes for the current trip and the state of the weather. More specifically there are four groups of variables:

1. Based on the users' past behaviour, which are calculated using as input past choices the user has made and the inferred behaviour as it is logged using the mobility patterns. These context variables include:

- "Too many car routes": We make use of a sliding-window based function that analyses the usage of car in terms of distance travelled over the previous time window period and compares it to the current time-window. In case an increase of car usage that exceeds a configured threshold is detected, the context variable is set to True.

- "Too many public transportation routes": We use a configurable sliding-window that compares the usage of public transportation in two subsequent periods. In case an increase of public transportation usage that exceeds a configured threshold is detected the context variable is set to True.

2. Based on the user's behaviour compared to other users.

- "Emissions increasing compared to others": The variable is set when the user's emissions as indicated by her/his past behaviour are increasing compared to other users. In order to set this variable we normalize the emissions of similar users and consider a configurable sliding window. When the emissions exceed a certain threshold compared to the mean value of the emissions of similar users the variable is set to true. The calculation of the emissions is based on a simple model 
that has been tested in real life conditions. The model is linear with respect to the distance covered with a specific means of transportation and provides a simplified estimation of the emissions caused. The underlying assumption for using a simplified model is that past research has provided evidence that exact and accurate emission values do not provide additional value when communicated to end-users (Brazil et al. 2015). This means that it is enough for the designer of a system to calculate and provide an estimate that communicates emissions' related information to end-users. The specific emission coefficients per transportation mode are as follows: Subway - 40 gr/km; Heavy Rail - 60 gr/km; Bus - 50 gr/km; Car - 100 $\mathrm{gr} / \mathrm{km}$. Based on trip characteristics, which are calculated using as input the available routes. These context variables are activate on a per route basis and include the following:

- "Walking Distance": It means that the destination is in a walking distance. We use as input the distance that needs to be covered in a route alternative and a configurable threshold. A global value of the threshold can be set by the system administrator while users can provide a personalized value, according to their preferences. In cases when the route distance is lower than the threshold the "walking distance" context variable is set to True.

- "Bike Distance": It means that the destination is in a biking distance. We use as input the distance that needs to be covered in a route alternative and a configurable threshold, similarly to the previous case. A global value of the threshold can be set by the system administrator while users can provide a personalized value, according to their preferences. In cases when the route distance is lower than the threshold the "bike distance" context variable is set to True.

- "Duration similar to driving": This context variable is activated when routes that are alternative to driving (including public transportation, bicycling and walking) are detected with a similar duration. In order to set the value of this context variable, the system compares the routes that do not involve the use of a car with those that have as a main modality the car.

3. Based on environmental information. In this case we make use of weather information and the context variable refers to the current status of the weather. The context variable is defined as follows:

- "Nice Weather": The variable is set to True when the temperature and precipitation levels exceed a certain configurable threshold. The threshold is set by the system administrator, however it can be configured on a per user level in order to provide personalized information. Since the content of some messages depends on the modes which are part of the route which those messages try to persuade the user to follow, different versions of messages have been created for the various modes (public transport, car, bike, bike sharing, walk, bike\&ride, park\&ride and car sharing). Therefore, each persuasive message can be associated to one or more transportation modes. Table 4 provides indicative examples of persuasive messages. The transportation mode(s) that each message tries to persuade the user to follow are depicted in brackets in Table 4. The messages may contain placeholders for percentages or numbers (bolded in Table 4). The actual values of the placeholders are calculated in the runtime through a number of functions that have been developed for that purpose. The calculation is based on the mode detection of user activities through the approach described in Section 4. 
Table 4 Indicative persuasive messages for different contexts and persuasive strategies

\begin{tabular}{llll}
\hline Context & Suggestion & Comparison & Self-monitoring \\
\hline Bike & It's not too far. Take your & PBikeSD \% of users & Last week you caused \\
distance & bike instead of car and & biked for similar & CO $_{2}$ Em g of $\mathrm{CO}_{2}$ \\
& reach your weekly goal & distances [Bike] & emissions. Try to reduce \\
& [Bike] & & it by biking [Bike] \\
Too many & Take public transport to & Take public transport. & Last week in PCar $\%$ \\
car routes & improve your C0 2 & PReduceDriving $\%$ of & of your routes you were \\
& footprint [Public & users have already reduced & using your car. Consider using \\
& Transport] & driving [Public Transport] & this route [Park\& ride] \\
Nice & Today it's sunny! Take the & PPtGW \% of users & When the weather was \\
weather & opportunity to combine & used public transport & good you used bike \\
& bike with public & when the weather was & sharing MinBikeSharing \\
& transportation to save $\mathrm{CO}_{2}$ & as good as today! & minutes per day on average \\
& [Public Transport] & [Bike Sharing]
\end{tabular}

\subsection{Message selection method}

The persuasive message selection method is outlined in Fig. 3. It takes into account user susceptibility to the different persuasive strategies, the current personal, trip and environmental context, as well as the transportation mode targeted. Given a pair of a user and a route as the target for user persuasion, one persuasive message is selected, on the basis of a)

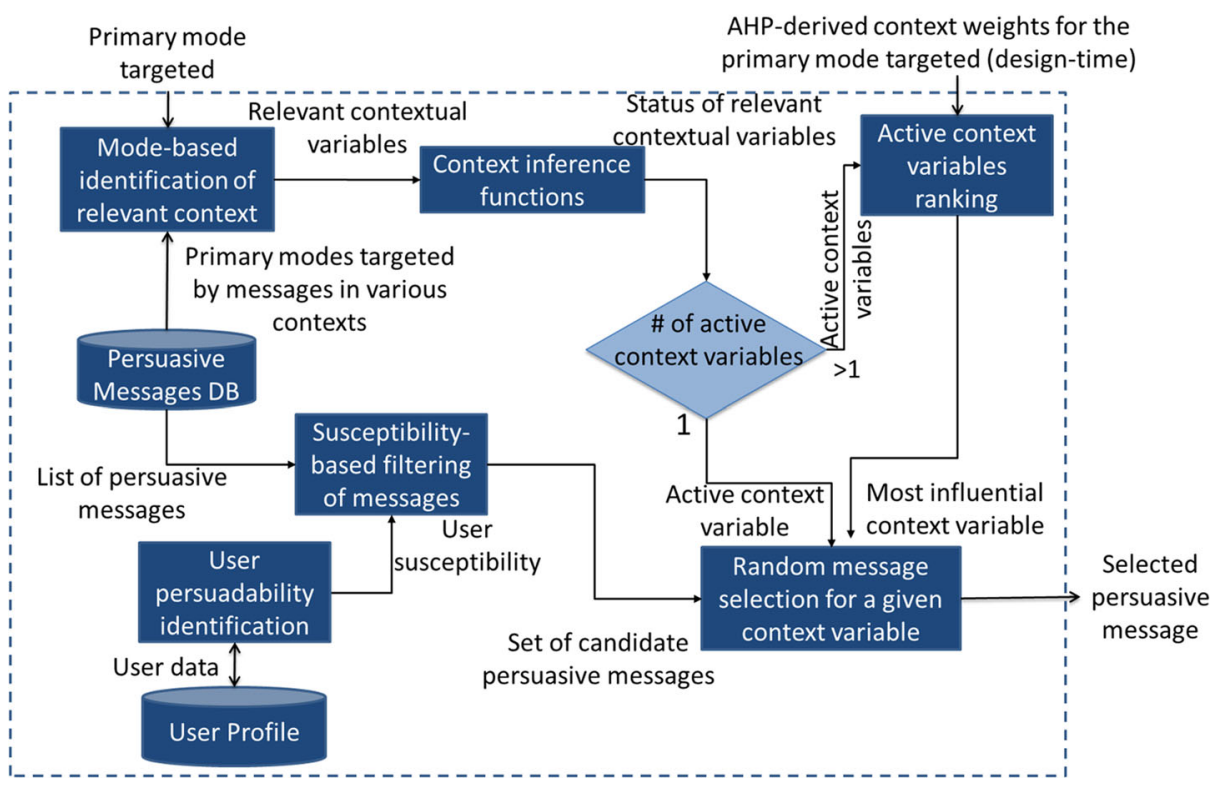

Fig. 3 Overview of the persuasive message selection process based on user susceptibility to the different persuasive strategies and the current context 
user susceptibility to the different persuasive strategies, and b) the current context. User persuadability actually defines the selection space, since a message is selected among the set of messages implementing the persuasive strategy that works best with the particular user. Since multiple messages have been defined per persuasive strategy, in order to select among the messages corresponding to a specific persuasive strategy, the current status of the various binary contextual variables for which context-aware messages have been defined, along with the primary transportation mode of the route that each message tries to persuade the user to follow, are taken into account. Since some primary transportation modes are only targeted by messages in particular contexts but not in others, some contextual variables may be irrelevant for a given primary mode. For example, the 'walking distance' context variable is irrelevant for all primary modes but walking. Therefore, the message selection method identifies the relevant contextual variables for a given primary mode (see also Fig. 3). There are two possibilities depending on the number of the relevant contextual variables that are active (i.e. their status is true): a) if only one contextual variable is true, a message targeting the given transportation mode is randomly selected among the one(s) defined for that context variable; b) if more than one contextual variables are true at the same time, there is a need to select a message defined for the context variable that is considered more influential.

In order to rank context variables according to their potential for influencing users, we developed a model based on the Analytic Hierarchy Process (AHP), a Multi Criteria Decision Making (MCDM) method that has been extensively studied, refined and applied since then in several application domains. More specifically, we asked three domain experts in Intelligent Transportation Systems to evaluate by pairwise comparisons the relative influence that the contextual variables have on the user choice of the corresponding mode. Experts were asked to rate the relative influence of each contextual variable compared to its pair on a nine-point scale, ranging from equal influence (1) to extreme relative influence (9). Their responses were used to derive the individual context weights for seven conflicting cases which we identified (these are cases where two or more context variables can be active at the same time). The individual weights were aggregated by using the AIP (Aggregation of Individual Priorities) method (Forman and Peniwati 1998). It should be noted that the AHP-based generation of the contextual variable weights for the conflicting cases is performed once at design time and then used during runtime to identify the most influential context variable for each case.

\section{Evaluation and discussion}

To evaluate our approach for sustainable transportation we setup a pilot study which allowed us to investigate the mobility behaviour, the travel mode choices as well as the attitudes and user experiences of participants. Moreover, the impact of our approach on user behaviour and attitudes and the effectiveness of persuasive messages to nudge users to make more sustainable mobility choices were examined. During the pilot study, users from the city of Vienna in Austria installed and used the OPTIMUM application to plan their everyday urban trips. Vienna is a city that offers a variety of mode choices including advanced public transportation networks as well as bike and car sharing options. The duration of the pilot study was 6 weeks, beginning on April 2017 and ending on May 2017. In total 30 participants took part in the pilot study, 15 females, 15 males, between 21 and 70 years (mean $=39.5$; $\mathrm{SD}=$ 12.04 ; median $=41.5)$. The mean age of female users was 35.73 years $(\mathrm{SD}=9.40$; median $=36)$ and of male users 43.27 ( $\mathrm{SD}=13.17$; median $=46)$. The overall procedure and data collection methodology are described in Table 5. Participants downloaded and installed 
Table 5 Overall procedure of our pilot study

\begin{tabular}{lll}
\hline Phase & Timing & Method \\
\hline Recruitment & 3 weeks before the pilot & Participant criteria catalogue \\
Start & Pre-interaction & App download, user registration \\
- & After 1 week & Pre-trial questionnaire \\
- & After 6 weeks & Post-trial questionnaire \\
End & After 7-8 weeks & Phone interviews \\
\hline
\end{tabular}

the OPTIMUM app from Google Store on their mobile phone. The subjects filled in a set of online questionnaires before and after the pilot and were interviewed at the end of the study. The pre-trial questionnaire asked for participants' travel preferences and past travel behaviour. The post-trial questionnaire contained questions regarding usage and user experience of the app, the usefulness of the persuasive interventions as well as their influence on participants' actual travel behaviour and environmental awareness. Finally, four semistructured interviews were carried via telephone after 7 weeks and lasted for about $30 \mathrm{~min}$ each. Topics covered during the interviews included the general impression of the app, user experience related topics, opinions about persuasive messages and their influence on travel mode choices and personal environmental awareness. Both quantitative and qualitative data were collected to gather as much insight as possible. For the data analysis, we took into account logged interactions of users using the OPTIMUM app, the mobility and the personality type of participants and responses to the questionnaires. In order to better understand the behaviour and usage patterns of the users several means to collect data regarding these aspects were implemented. Data collection and analysis focused on the persuasive messages data and the GPS data. Specifically, whenever the user searched for a possible route with the app, the requests were collected and logged over the time of the pilot, thereby providing us the possibility to analyse users' activity with regard to their route requests. In addition, the app automatically recorded the GPS-tracks and sensor information of the users' phones, and this data was then used to automatically impute the travel modality of the users.

\subsection{Results}

During the pilot study, 182 routing requests were sent by the users and 175 messages were displayed (matching corresponding context activations). The effectiveness of the messages was measured by considering two cases as having a positive effect: i) "popup only": the user provided a positive reply in the related "popup" displayed in the route selection screen (see part 2 of Fig. 2) and ii) "popup and viewed routes": the user provided a positive reply in the "popup" as in case (i) or the user checked the details screen of the recommended route to which the message referred (the route details appear when the users selects a route and provides details regarding the itinerary). Note that we report case (ii) because there were cases where users skipped the "popup" and didn't provide any feedback (by e.g. pressing the "home" button of their smartphone). In more details, we received feedback from the "popup" for 51 requests. The positive feedback was for 15 messages or $30 \%$ of the total messages for which we received feedback. When considering also the viewed routes (as described in case (ii) above) the positive feedback was $40 \%$ although we cannot be certain that users who provided positive feedback actually followed the more environmentally friendly route. 
Moreover, we analysed the collected questionnaire responses from 27 users ( 3 users did not fill the online questionnaires) and interview data in order to identify the effect of the persuasive interventions on user mobility choices. Subjects stated that they found the messages useful. Most of them reported that the ranking of routes influences their decision regarding the transport choice (see part 1 of Fig. 4). Participants reported that the messages are easy to understand and clear (see part 2 of Fig. 4). Moreover, the willingness to see persuasive messages in their daily mobile applications was high as participants stated that they would like to receive persuasive messages in route planning applications (see part 3 of Fig. 4), while the persuasiveness of the personalized messages was perceived as somewhat convincing as shown in part 4 of Fig. 4. The perceived quality and ease of understanding of the interventions followed the same patterns in the interviews. Two participants recalled an example of transport modes that they were persuaded by the app to follow "Received the better option to take the bus instead of the tramway" - "Received better route to go the doctor". Moreover, a participant stated "I received the message: Nice weather, others have been walking. At that moment, I changed my mind and walked down the Mariahiflerstrasse instead of taking the underground (U3) to the station Volkstheater". In the phone interview, one participant shared her thoughts that she personally had become more aware of the possibility to use the bike (although preferably not in city traffic). As a consequence, she used the bike two times to reach the train station instead of using public transport. Another interesting finding came out of users who stated that the suitability of a suggested mode would strongly depend on the given situation such as trip purpose and social context of the trip. Accordingly, the assumption holds that individual trip purpose has a strong influence on transport mode choice. This means that the personalization of messages can be improved by taking into account the trip purpose.

\subsection{Discussion}

In this section, we discuss the results obtained from the evaluation of our approach as well as the impact and the effectiveness of persuasive interventions. The effect of persuasive technologies can be measured by several ways. The most obvious outcome measured is the behavioural effect, i.e. the impact of persuasive technologies on behavioural change. Commonly this is measured by monitoring users' behaviour before and after the use of the persuasive technologies in order to understand whether users change their actions and behaviour or not. Data which are used to infer changes in users' behaviour include selfreported activity logs, GPS logs, cell phone tracking over the mobile network as well as
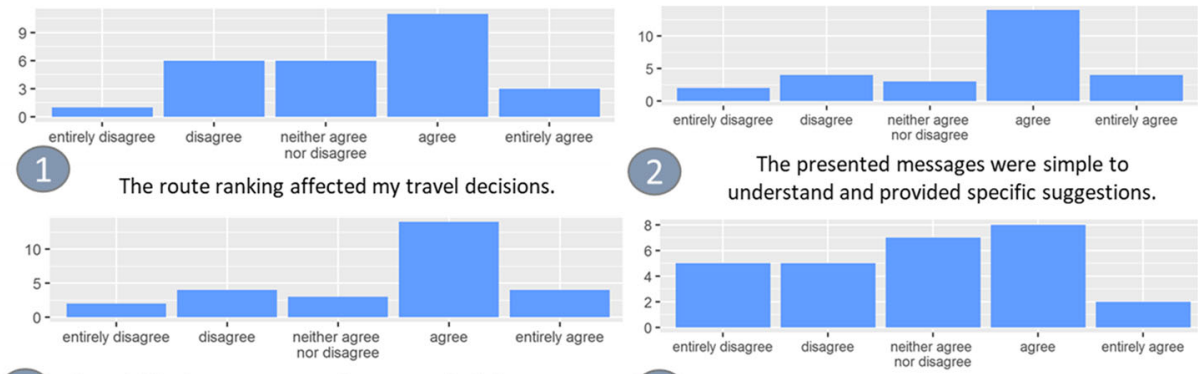

3 I would like to see messages to urge me to follow more environmentally friendly routes in route planning applications

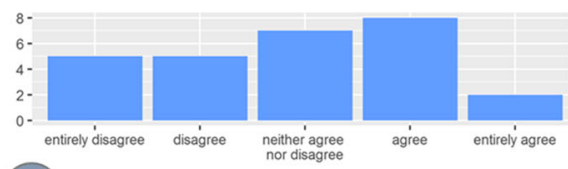

4 The persuasive messages were convincing.

Fig. 4 Study participants' responses to our online questionnaire 
qualitative data from interviews with users who provide subjective opinions and statements related to behaviour changes. Focusing on measuring behavioural changes is probably the most accurate method to evaluate the impact of the persuasive interventions. However, it is not always possible to measure or accurately determine actual behavioural change. The main reason is the limited usage period of the persuasive technologies. A usage period of a few weeks is not sufficient to understand the effect of an intervention, and in the transportation domain where travel habits can be difficult to change, the case becomes even harder.

For these reasons, researchers measure the impact of persuasive technologies using metrics such as qualitative questionnaires that identify the acceptance of the technologies, the attitude change with respect to environmentally friendly transport modes and the perceived persuasive effects. A recent review of persuasive applications for sustainable mobility (Sunio and Schmöcker 2017) reports that out of nine applications reviewed only three attempt to evaluate the effectiveness of behavioural change interventions. For the three applications (namely QT, Peacox and SuperHub) the main findings concern changes in attitude change and intention to change to environmentally friendly transport modes. Our quantitative feedback data provide insight on users' decisions at the point when they select a route, an approach that has not been used in previous studies. The analysis of the results showed that $40 \%$ of persuasive messages received positive feedback (i.e. users indicated that the message had some effect on their route choice). Although we cannot be sure that users' feedback actually led to decisions of green transport modes in all cases, even small improvements of 3\%-5\% of decisions for green transport modes can provide significant support to targets set in sustainable urban mobility plans (SUMPs) devised by cities all around Europe (for example the SUMP of the city of Vienna targets a mode share of $80 \%$ of eco-mobility and $20 \%$ of car traffic by 2025 compared to a $72: 28$ in 2013).

In addition, we observed that most context variables were not activated because of limited use of the GPS tracking functionality, which could be turned off by users in an opt-out manner. This created a bias towards the presentation of messages with contextual variables in the categories of trip characteristics and environment, rather than in that of personal characteristics, since in contrast to the former, the context variables of the latter category are activated based on the analysis of tracking data. For example, messages with the context "Nice Weather", were displayed more frequently than those with the context "Emissions Increasing". Note that the limited use of tracking was mostly due to battery consumption problems which is proven to be a problem in such applications. The main goal of our approach is to support behaviour change of users towards more sustainable travel options. The results from our pilot show evidence that the implemented persuasive strategies had some impact on users' behaviour. However, users' feedback provided hints for future work: ranking of routes in the app was perceived as having an influence on the route decisions, so the presentation of the route that is considered as the target for the persuasive attempts in the first place of the list should be considered in our future work. Also, the messages were perceived as useful, and work in the future should focus on how to further improve and personalize them in order to achieve a higher impact. Moreover, since most of the users participated in the pilot study were already environmental friendly, the potential impact of persuasive messages could not be demonstrated in its full potential. Furthermore, the fairly short pilot timeframe failed to capture important aspects such as seasonality and the effectiveness of the approach on a longer-term basis. In the future, we aim to conduct a larger scale and longer term pilot study that will focus more on car drivers that have a positive attitude towards environmental friendly modes. Specifically, we plan to extend our evaluation with users classified as Potential non-Drivers, in terms of their mobility type, as defined in Section 5. These include people that drive but don't really like driving, they are keen to drive 
less but still prefer the car to cycling, while they also see problems with public transport, people that would like to cut down on car use and agree that the bus can be quicker, but still see problems with using public transport, and people that only use the car when necessary and believe cars reduce quality of life. This will allow us to address the aforementioned bias towards the presentation of messages with contextual variables of specific categories.

Last but not least, we would like to mention that the city of Vienna can be considered as an optimal testbed for approaches such as ours as it provides a highly reliable, accessible and dense transportation network with manifold eco-friendly options. The assumption for a successful behavioural change system is the availability and accessibility of green transportation alternatives. Practically, this means that the chances of asking a traveller who lives in an area without public transport to switch to public transport are potentially non-existent. Besides modes' availability, the barriers in the transport options of the area where the intervention is deployed should be minimum, including the reliability of the transport system (e.g. frequent service interruptions can deter travellers from using public transportation), the ease of accessing the modes (e.g. e-tickets, accessible bus stops, location of bike sharing infrastructure, etc.). Our view is that the deployment of persuasive interventions should be part of a general transport planning approach, in cooperation with the transport authorities and various transport modes operators. An integrated approach where the transport system works for the benefit of travellers and persuasive technologies support travellers' decisions could provide significant impact.

\section{Conclusions and future work}

In this paper, we presented our approach for personalized behavioural change support by leveraging travel behaviour and personality profiles. Moreover, we described the results of a pilot study related to behavioural change interventions for sustainable urban mobility. The results showed evidence that our proposed approach had some impact on motivating users on a personal level to change their mobility behaviour and make more sustainable choices. For the future also improved and additional means for studying the actual travel modalities should be implemented. On an organizational level, it should be ensured that test participants are encouraged to allow the continuous tracking of their travel data. On a technical level battery consumption (the main factor for disallowing tracking) should be addressed, and also the quality of the data analysis should be improved as far as possible. Additionally, on a methodological level, travel behaviour should also be sampled more frequently using traditional means, e.g. sampling questionnaires or in-app feedback. Based on the results of our evaluation we will optimize the persuasive interventions services. Moreover, we are examining ways which could extend the proposed persuasive services. These include the implementation of visual (in addition to verbal) presentation of persuasive strategies (e.g. through graphs), that will allow us to examine the combined effect of visual and verbal presentations on users' behaviour and compare it to that of solely visual or verbal presentations. Examples include graphs that provide visual cues of the $\mathrm{CO}_{2}$ emissions caused by a user's activities compared to those of the other users (comparison strategy) and graphs that show the $\mathrm{CO}_{2}$ emissions caused by the user in different time periods (self-monitoring strategy). Furthermore, we are looking into ways of extending the contextual variables and integrate knowledge that concerns users' next activity. Towards this direction, the mode detection module is being extended to generate predictions of the users' next location. For example, if the next location is a shopping mall there is a high probability that the user is travelling for leisure, information that can be used in order to intensify the persuasive attempts. The 
knowledge of the users' next activity can also be helpful for providing proactive notifications that nudge them, for example, to take the bus when it passes by a station that is near the user.

Acknowledgments Research reported in this paper has been partially funded by the European Commission project OPTIMUM (H2020 grant agreement No. 636160-2) and the European Commission project MaaS4EU (H2020 grant agreement No. 723176).

Open Access This article is distributed under the terms of the Creative Commons Attribution 4.0 International License (http://creativecommons.org/licenses/by/4.0/), which permits unrestricted use, distribution, and reproduction in any medium, provided you give appropriate credit to the original author(s) and the source, provide a link to the Creative Commons license, and indicate if changes were made.

\section{References}

Anable, J. (2005). Complacent car addicts or aspiring environmentalists. Identifying travel behaviour segments using attitude theory. Transport Policy, 12(1), 65-78.

Anagnostopoulou, E., Magoutas, B., Bothos, E., Schrammel, J., Orji, R., Mentzas, G. (2017). Exploring the links between persuasion, personality and mobility types in personalized mobility applications. In International conference on persuasive technology (pp. 107-118). Cham: Springer.

Bond, D.S., Thomas, J.G., Raynor, H.A., Moon, J., Sieling, J., Trautvetter, J., Leblond, T., Wing, R.R. (2014). B-MOBILE-a smartphone-based intervention to reduce sedentary time in overweight/obese individuals: a within-subjects experimental trial. PloS One, 9(6), e100821. https://www.ncbi.nlm.nih.gov/pubmed/ 24964010.

Bothos, E., Mentzas, G., Prost, S., Schrammel, J., Röderer, K. (2014). Watch your emissions: persuasive strategies and choice architecture for sustainable decisions in urban mobility. Psychology Journal, 12(3), $107-126$.

Brazil, W., Caulfield, B., Bothos, E. (2015). Transport emissions information: lessons from the PEACOX project. ITRN 2015.

Chen, K.Y., Shah, R.C., Huang, J., Nachman, L. (2017). Mago: mode of transport inference using the halleffect magnetic sensor and accelerometer. Proceedings of the ACM on Interactive, Mobile, Wearable and Ubiquitous Technologies, 1(2), 8.

Collis, J.M. (1997). The OCEAN personality inventory: administrator's guide and test manual. University of Plymouth, Human Assessment Laboratory.

Fogg, B.J. (2002). Persuasive technology: using computers to change what we think and do. Morgan Kaufmann, 2002 (December). https://www.amazon.com/Persuasive-Technology-Computers-InteractiveTechnologies/dp/1558606432. Accessed 20 September 2018.

Forman, E., \& Peniwati, K. (1998). Aggregating individual judgments and priorities with the analytic hierarchy process. European Journal of Operational Research, 108(1), 165-169.

Fortuna, B., Rupnik, J., Brank, J., Fortuna, C., Jovanoski, V., Karlovcec, M., Leban, G. (2014). Qminer: data analytics platform for processing streams of structured and unstructured data. In Software engineering for machine learning workshop, neural information processing systems.

Franklin, V.L., Waller, A., Pagliari, C., Greene, S.A. (2006). A randomized controlled trial of Sweet Talk, a text-messaging system to support young people with diabetes. Diabetic Medicine, 23(12), 1332-1338.

Froehlich, J., Dillahunt, T., Klasnja, P., Mankoff, J., Consolvo, S., Harrison, B., Landay, J.A. (2009). UbiGreen: investigating a mobile tool for tracking and supporting green transportation habits. In Proceedings of the SIGCHI conference on human factors in computing systems (pp. 1043-1052). ACM. ISO 690.

Fukuoka, Y., Komatsu, J., Suarez, L., Vittinghoff, E., Haskell, W., Noorishad, T., Pham, K. (2011). The $\mathrm{mPED}$ randomized controlled clinical trial: applying mobile persuasive technologies to increase physical activity in sedentary women protocol. BMC Public Health, 11(1), 933.

Harries, T., Eslambolchilar, P., Stride, C., Rettie, R., Walton, S. (2013). Walking in the wild-Using an always-on smartphone application to increase physical activity. In IFIP conference on human-computer interaction (pp. 19-36). Berlin: Springer.

Hemminki, S., Nurmi, P., Tarkoma, S. (2013). Accelerometer-based transportation mode detection on smartphones. In Proceedings of the 11th ACM conference on embedded networked sensor systems (p. 13): ACM. 
Hong, Y., Dahlke, D.V., Ory, M., Hochhalter, A., Reynolds, J., Purcell, N.P., Eugene, N. (2013). Designing iCanFit: a mobile-enabled Web application to promote physical activity for older cancer survivors. JMIR Research Protocols, 2(1), e12. https://www.ncbi.nlm.nih.gov/pubmed/2361205.

Kang, J.H., Welbourne, W., Stewart, B., Borriello, G. (2004). Extracting places from traces of locations. In Proceedings of the 2nd ACM international workshop on wireless mobile applications and services on WLAN hotspots (pp. 110-118). ACM.

Kaptein, M. (2011). Adaptive persuasive messages in an e-commerce setting: the use of persuasion profiles. In ECIS (p. 183).

Lamsfus, C., Wang, D., Alzua-Sorzabal, A., Xiang, Z. (2015). Going mobile: defining context for on-the-go travelers. Journal of Travel Research, 54(6), 691-701.

Lee, S., Lee, J., Lee, K. (2017). VehicleSense: a reliable sound-based transportation mode recognition system for smartphones. In 2017 IEEE 18th international symposium on a world of wireless, mobile and multimedia networks (WoWMoM) (pp. 1-9). IEEE.

Mizell, D. (2003). Using gravity to estimate accelerometer orientation. In ISWC '03, Proceedings of the 7th IEEE international symposium on wearable computers (p. 252). Washington, DC: IEEE Computer Society.

Oinas-Kukkonen, H. (2013). A foundation for the study of behavior change support systems. Personal and Ubiquitous Computing, 17(6), 1223-1235.

Oinas-Kukkonen, H., \& Harjumaa, M. (2008). A systematic framework for designing and evaluating persuasive systems. In Persuasive technology (pp. 164-176).

Pedregosa, F., Varoquaux, G., Gramfort, A., Michel, V., Thirion, B., Grisel, O., Blondel, M., Prettenhofer, P., Weiss, R., Dubourg, V. (2011). Scikit-learn: machine learning in python. Journal of Machine Learning Research, 12, 2825-2830.

Purpura, S., Schwanda, V., Williams, K., Stubler, W., Sengers, P. (2011). Fit4life: the design of a persuasive technology promoting healthy behavior and ideal weight. In Conference on human factors in computing systems (pp. 423-432). ACM.

Rammstedt, B., \& John, O.P. (2007). Measuring personality in one minute or less: a 10-item short version of the Big Five Inventory in English and German. Journal of Research in Personality, 41(1), 203-212.

Reddy, S., Mun, M., Burke, J., Estrin, D., Hansen, M., Srivastava, M. (2010). Using mobile phones to determine transportation modes. ACM Transactions on Sensor Networks (TOSN), 6(2), 13.

Reitberger, W., Ploderer, B., Obermair, C., Tscheligi, M. (2007). The percues framework and its application for sustainable mobility. Persuasive Technology, LNCS, 4744, 92-95.

Rodgers, A., Corbett, T., Bramley, D., Riddell, T., Wills, M., Lin, R.B., Jones, M. (2005). Do u smoke after txt? Results of a randomised trial of smoking cessation using mobile phone text messaging. Tobacco Control, 14(4), 255-261.

Senožetnik, M., Bradeško, L., Kažic, B., Mladenic, D., Šubic, T. (2016). Spatio-temporal clustering methods. In Proceedings of the 19th international multiconference information society - IS 2016, Ljubljana, Slovenia, volume D.

Shafique, M.A., \& Hato, E. (2015). Use of acceleration data for transportation mode prediction. Transportation, 42(1), 163-188.

Sunio, V., \& Schmöcker, J.D. (2017). Can we promote sustainable travel behavior through mobile apps? Evaluation and review of evidence. International Journal of Sustainable Transportation, 11(8), 553-566.

Torning, K., \& Oinas-Kukkonen, H. (2009). Persuasive system design: state of the art and future directions. In Persuasive technology (p. 30). ACM.

Urbančič, J., Bradeško, L., Senoöetnik, M. (2016). Near real-time transportation mode detection based on accelerometer readings. In Proceedings of the 19th international multiconference information society IS 2016, Ljubljana, Slovenia, volume D.

Wiafe, I., \& Nakata, K. (2012). Bibliographic analysis of persuasive systems: techniques; methods and domains of application. In Persuasive technology: design for health and safety; PERSUASIVE:2012; Linköping; Sweden (No. 068, pp. 61-64).

Widhalm, P., Nitsche, P., Brändie, N. (2012). Transport mode detection with realistic smartphone sensor data. In 201221 st international conference on pattern recognition (ICPR) (pp. 573-576). IEEE. 